\title{
ChemComm
}

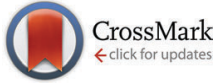

Cite this: Chem. Commun., 2015 51,17136

Received 21st July 2015,

Accepted 29th September 2015

DOI: $10.1039 / c 5 c c 06094 j$

www.rsc.org/chemcomm

\section{Bright fluorogenic squaraines with tuned cell entry for selective imaging of plasma membrane vs. endoplasmic reticulum $\dagger$}

\author{
Mayeul Collot, ${ }^{\star a}$ Rémy Kreder, ${ }^{a}$ Anatoliy L. Tatarets, ${ }^{b}$ Leonid D. Patsenker, ${ }^{b}$ \\ Yves Mely ${ }^{a}$ and Andrey S. Klymchenko*a
}

A rational design of squaraine dyes with lipophilic and zwitterionic groups tunes cell entry, allowing for selective far-red/near-infrared imaging of plasma membrane vs. endoplasmic reticulum. They exhibit up to 110 -fold fluorescence enhancement in biomembranes and enable cellular imaging at $1 \mathrm{nM}$ concentration, which make them the brightest membrane probes to date.

Fluorescence imaging of living cells is a rapidly expanding research direction. It relies on the development of fluorescent probes ${ }^{1}$ which are smart fluorescent tools built from organic molecules, proteins ${ }^{2}$ or nanoparticles. ${ }^{3}$ Of particular interest are probes for cell plasma membranes, because the latter delimit the cellular contour and thus enable a clear distinction between extra- and intracellular components. In addition to the basic function of the cell barrier, membranes are the turntables for crucial processes in neurobiology, muscle contraction, and cell signalling. ${ }^{4}$

Fluorescently labelled lectins, notably wheat germ agglutinin (WGA), are among the most popular fluorescent membrane probes. ${ }^{5}$ On the other hand, membrane probes based on small organic dyes, being less expensive and more chemically stable, present the unique possibility of being precisely located and oriented in the lipid bilayer, ${ }^{6}$ which are important for studies of the lateral lipid organization of biomembranes (lipid rafts), ${ }^{7-9}$ FRET with membrane proteins ${ }^{10}$ and super-resolution imaging. ${ }^{11,12}$ However, the widespread use of these probes is limited by their poor performance compared to labelled WGA. For instance, the well-known FM 4-64 internalizes rapidly in cells by endocytosis and its brightness is low. ${ }^{13,14}$ Long-chain cyanines, such as DiO, DiI, DiD, and DiR, due to poor solubility, stain plasma membranes inefficiently with a large amount of intracellular

\footnotetext{
${ }^{a}$ Laboratoire de Biophotonique et Pharmacologie, UMR 7213 CNRS, Université de Strasbourg, Faculté de Pharmacie, 74, Route du Rhin, 67401, Illkirch, France. E-mail: andrey.klymchenko@unistra.fr, mayeul.collot@unistra.fr

${ }^{b}$ The State Scientific Institution "Institute for Single Crystals" of the National Academy of Sciences of Ukraine, 60, Lenin Ave., Kharkiv, 61001, Ukraine

$\dagger$ Electronic supplementary information (ESI) available: All experimental protocols and additional cellular data. See DOI: 10.1039/c5cc06094j
}

dotted fluorescence. ${ }^{15,16}$ Remarkably, cell staining with FM 4-64 or the cyanine probes requires 1-5 $\mu \mathrm{M}$ concentration, which is $\sim 100$-fold larger than the concentration theoretically needed to achieve a probe/plasma membrane lipid ratio of $1 / 100$ (for $10^{6}$ cells per $\mathrm{mL}$ ). Above this ratio, the probe may significantly perturb the biomembrane properties. Examples of highly specific plasma membrane probes working at low concentrations with long retention times appeared rather recently, notably using 3-methoxyflavones, ${ }^{17}$ Nile Red, ${ }^{18}$ and BODIPY. ${ }^{19,20}$ However, high-performance membrane probes in the far-red to near-infrared (NIR) regions are still missing. Such probes should be particularly interesting for both in vitro and in vivo imaging applications $^{21}$ as well as for multi-colour imaging with the green and red fluorescent markers commonly used in biology. In this respect squaraine dyes are particularly attractive due to their absorption and emission in the far-red to NIR regions, exceptional molar extinction coefficients (around $300000 \mathrm{M}^{-1} \mathrm{~cm}^{-1}$ ) and high quantum yields, which made them very popular for the development of fluorescent probes. ${ }^{22-27}$ However, so far squaraines have never been used to build a probe selective to cell plasma membranes.

Herein, new squaraine-based membrane probes were designed with a long hydrocarbon chain and a zwitterionic group, which serve as anchors to fix the fluorophore at the interface and prevent the flip-flop of the probes between the leaflets, as we have shown earlier for 3-hydroxyflavone ${ }^{28}$ and Nile Red dyes. ${ }^{18}$ First, SQ8S was designed, where the squaraine dye bears a hydrophobic tail at one end and a zwitterionic polar head at the other end (Scheme 1). SQ8S was synthesised from the unsymmetrical squaraine 2 , which was coupled to $N, N$-dimethylpropane-1,3-diamine and was subsequently reacted with 1,3-propane sultone (Scheme 1). In a second approach, SQ12S and dSQ12S were designed to bear the fluorophore and respectively one or two amphiphilic anchors. These anchors were composed of a hydrocarbon chain (C12) and an ammonium sulphate inner salt as a zwitterionic moiety, which should favour high affinity to the lipid membrane. SQ12S and dSQ12S were obtained from a block synthesis based on CuAAC click chemistry. ${ }^{29}$ The key amphiphilic zwitterionic 

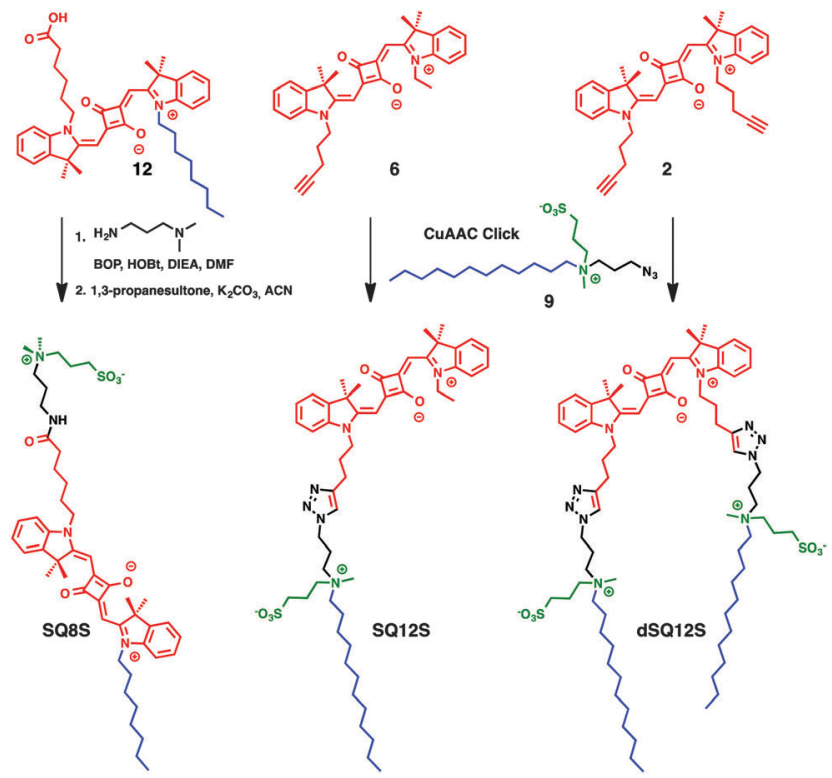

Scheme 1 Structures and synthesis strategy of membrane probes consisting of a squaraine dye (red), a hydrocarbon chain (blue) and a zwitterionic moiety (green).

block 9 was obtained by reacting $N$-methyldodecylamine with 1-azido-3-chloropropane followed by quaternisation with 1,3-propanesultone (Scheme S2, ESI $\dagger$ ). Block 9 was then clicked to the unsymmetrical and symmetrical squaraines $\mathbf{6}$ and $\mathbf{1 2}$ (Scheme S1, ESI $\dagger$ ) to respectively obtain SQ12S and dSQ12S (Scheme 1).

In order to investigate the ability of these fluorophores to probe lipophilic environments, spectroscopic analyses were performed in dioxane, methanol, DMSO, and phosphate buffer (20 mM, pH 7.4) and in Large Unilamelar Vesicles (LUVs) composed of 1,2-dioleoyl-sn-glycero-3-phosphocholine (DOPC). These data were systematically compared to a dye of similar colour: 1,1'-dioctadecyl-3,3,3',3'-tetramethylindodicarbocyanine perchlorate (DiD), a commercially available plasma membrane tracer, consisting of cyanine- 5 bearing two C-18 hydrocarbon chains. ${ }^{16}$ In organic solvents, the new dyes showed sharp absorption and emission bands with a quantum yield (QY) reaching 70\% in apolar dioxane (Table 1). The QY values in methanol were significantly lower, which, in line with our earlier studies, ${ }^{25}$ show that squaraine dyes undergo some quenching in polar protic solvents. All new squaraine dyes showed very low fluorescence in water, while for DiD it was not even detectable. Their absorption spectra were very broad (Fig. 1), suggesting that in aqueous buffer the dyes form non-fluorescent aggregates. Upon binding to lipid membranes, squaraine probes showed a strong increase in fluorescence intensity with QY ranging between 49 and 70\%. The absorption spectra suggested that in the presence of LUVs the dye aggregates were disrupted, so that the highly emissive molecular form was inserted into the bilayers. Importantly, imaging giant vesicles with polarized two-photon laser excitation revealed that all three probes presented parallel orientation with respect to the bilayer plane (Fig. 2, Fig. S4, ESI $\dagger$ ), which was originally expected only for dSQ12S bearing two anchor groups.
Table 1 Photo-physical properties of the NIR membrane probes. QY is the fluorescence quantum yield

\begin{tabular}{|c|c|c|c|c|c|}
\hline Probe & Solvent & $\begin{array}{l}\lambda_{\text {Abs max }} \\
(\mathrm{nm})\end{array}$ & $\begin{array}{l}\varepsilon \text { at } \lambda_{\mathrm{Abs} \max } \\
\left(\mathrm{M}^{-1} \mathrm{~cm}^{-1}\right)\end{array}$ & $\begin{array}{l}\lambda_{\text {Em max }} \\
(\mathrm{nm})\end{array}$ & $\begin{array}{l}\text { QY } \\
(\%)\end{array}$ \\
\hline \multirow[t]{5}{*}{ DiD } & Dioxane & 644 & 210000 & 664 & 80 \\
\hline & Methanol & 644 & 250000 & 664 & $33^{d}$ \\
\hline & DMSO & 650 & 276000 & 670 & 62 \\
\hline & Phosphate buffer ${ }^{a}$ & 592 & 60800 & $\mathrm{~N} / \mathrm{A}^{c}$ & $\mathrm{~N} / \mathrm{A}^{c}$ \\
\hline & LUVs DOPC $^{b}$ & 650 & 210000 & 671 & 13 \\
\hline \multirow[t]{5}{*}{ SQ8S } & Dioxane & 639 & 267000 & 648 & 67 \\
\hline & Methanol & 631 & 300000 & 637 & 15 \\
\hline & DMSO & 646 & 233000 & 654 & 46 \\
\hline & Phosphate buffer ${ }^{a}$ & 628 & 166300 & 636 & 2.2 \\
\hline & LUVs DOPC $^{b}$ & 640 & 305000 & 647 & 70 \\
\hline \multirow[t]{5}{*}{ SQ12S } & Dioxane & 638 & 272000 & 646 & 57 \\
\hline & Methanol & 629 & 296000 & 637 & 10 \\
\hline & DMSO & 644 & 211000 & 653 & 40 \\
\hline & Phosphate buffer ${ }^{a}$ & 626 & 188600 & 634 & 1.4 \\
\hline & LUVs DOPC $^{b}$ & 640 & 314000 & 647 & 67 \\
\hline \multirow[t]{5}{*}{ dSQ12S } & Dioxane & 642 & 285000 & 649 & 73 \\
\hline & Methanol & 632 & 304000 & 640 & 17 \\
\hline & DMSO & 646 & 215000 & 654 & 63 \\
\hline & Phosphate buffer ${ }^{a}$ & 640 & 108000 & 637 & 0.46 \\
\hline & LUVS DOPC $^{b}$ & 642 & 274000 & 649 & 49 \\
\hline
\end{tabular}

${ }^{a} 20 \mathrm{mM}, \mathrm{pH} 7.4 .^{b} 200 \mu \mathrm{M}$ DOPC in phosphate buffer (20 mM, pH 7.4). ${ }^{c}$ No fluorescence was observed. ${ }^{d} \mathrm{DiD}$ in $\mathrm{MeOH}$ was used as a reference for the quantum yield $(\mathrm{QY})$ measurements. ${ }^{30}$
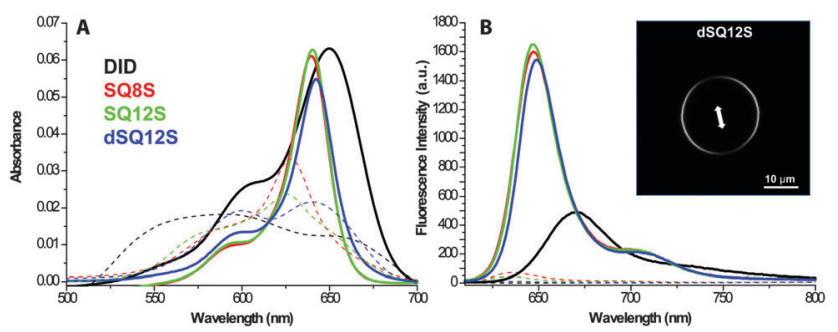

Fig. 1 Absorption (A) and emission (B) spectra of the membrane probes in phosphate buffer (20 mM pH 7.4, dashed lines) and in the presence of $200 \mu \mathrm{M}$ DOPC vesicles (solid lines). The concentration of probes was $200 \mathrm{nM}$. The inset shows the two-photon excited fluorescence image of a giant vesicle (DOPC) stained with dSQ12S showing the preferential orientation of the probe in the bilayer.

On the other hand, DiD failed to reach high quantum efficiency when added to LUVs (QY $=13 \%)$. We suppose that the direct addition of DiD from DMSO solution to an aqueous suspension of LUVs produced partial dye precipitation and thus inefficient binding to lipid membranes. Overall, the fluorogenic response of the new amphiphilic squaraines to lipid vesicles made these dyes promising as probes for biological membranes.

First, kinetic cell binding studies were performed, where the probes were added to a suspension of lymphocytes (Fig. S5, ESI $\dagger$ ). Whereas the DiD probe did not exhibit any significant fluorescence enhancement in the presence of cells, the squaraine probes displayed a burst of fluorescence intensity in the first seconds. It was noteworthy that SQ8S and SQ12S rapidly reached the plateau, while the fluorescence intensity of dSQ12S increased slowly, probably due to a slower de-aggregation and membrane binding process. The emission spectra of the probes recorded 


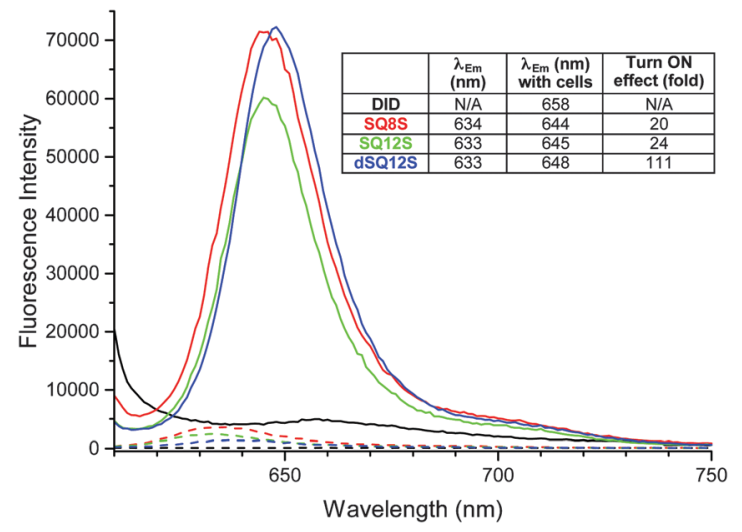

Fig. 2 Fluorescence spectra of membrane probes in buffer (dashed lines) and in the presence of lymphocytes $\left(10^{6}\right.$ cells per $\mathrm{mL}$ ) after $15 \mathrm{~min}$ (plain lines). Inset: Emission wavelengths and fluorescence enhancement in the presence of cells provoked by the insertion of the probes in the membranes.

after 15 min incubation with lymphocytes (Fig. 2) showed that DiD failed to stain efficiently the cells, whereas the squaraine-probes displayed an important fluorescence enhancement of 20-24-fold for SQ8S and SQ12S and up to 111-fold for dSQ12S, which are in good agreement with the results of DOPC vesicles (Table 1).

The ability of the new probes to stain cell membranes was then investigated by laser scanning confocal fluorescence microscopy using an adherent cell line (HeLa). HeLa cells were incubated with the squaraine probes at a concentration of $20 \mathrm{nM}$ without the washing step and imaged at different times (Fig. S6, ESI $\dagger$ ).

Even after 5-10 min of incubation squaraine probes displayed bright fluorescence in cell membranes with no background fluorescence (Fig. 3). Despite similarities in their structures and spectral properties, the new probes presented notable differences in their internalization and distribution within cells.
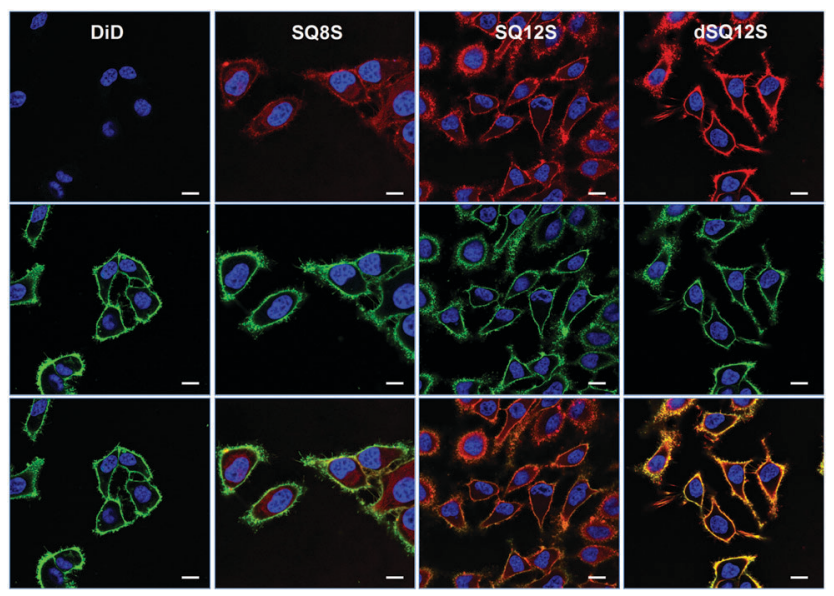

Fig. 3 Laser scanning confocal microscopy images of HeLa cells incubated for 5-10 minutes with the DID or squaraine probes at $20 \mathrm{nM}$ without washing (red). Nucleus was stained by Hoechst $\left(2 \mu \mathrm{g} \mathrm{mL}{ }^{-1}\right.$ ) (blue) and the plasma membrane was stained with WGA-488 (5 $\mathrm{g} \mathrm{mL}^{-1}$ ) (green). Top: images recorded with the probes and Hoechst channels, middle: images with the WGA-488 and Hoechst channels and bottom: merge of the three channels. For imaging squaraine and DiD probes, the excitation wavelength was $632 \mathrm{~nm}$, while the emission was set between 640 and $750 \mathrm{~nm}$. Scale bar is $10 \mu \mathrm{m}$.

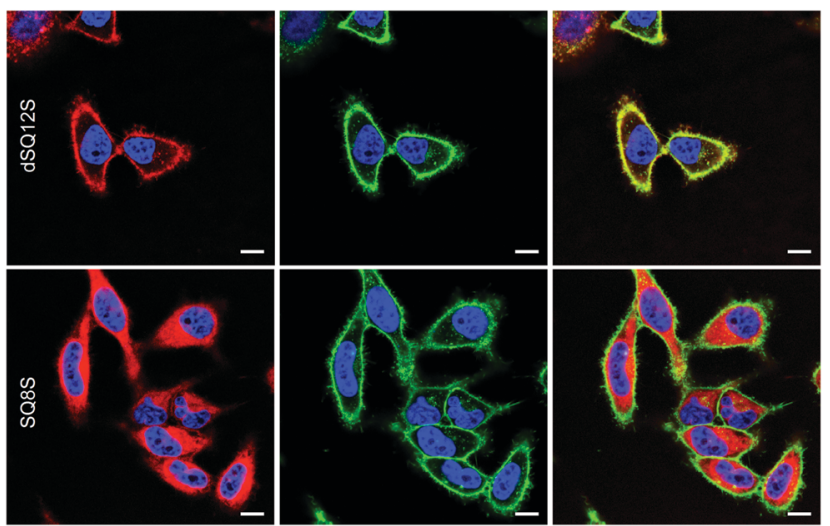

Fig. 4 Laser scanning confocal microscopy images of HeLa cells incubated for $1 \mathrm{~h}$ with $1 \mathrm{nM}$ dSQ12S (top images) and SQ8S (bottom) without washing (red). Nucleus was stained with Hoechst $\left(2 \mu \mathrm{g} \mathrm{mL}^{-1}\right)$ (blue) and the plasma membrane was stained with WGA-488 (5 $\mathrm{g} \mathrm{mL}^{-1}$ ) (green). Left: images recorded with the squaraine and Hoechst channels, middle: images recorded with the WGA-488 and Hoechst channels and right: merge of the three channels. For imaging squaraine probes, the excitation wavelength was $632 \mathrm{~nm}$, while the emission was set between 640 and $750 \mathrm{~nm}$. Scale bar is $10 \mu \mathrm{m}$.

Colocalisation experiments with WGA-488, a commercially available green membrane marker, showed that dSQ12S stained well the plasma membrane, whereas SQ8S accumulated rapidly in the cytoplasm. SQ12S had an intermediate behaviour as it was distributed both on the plasma membrane and the cytoplasm. Taking advantage of their exceptional brightness, the squaraine probes used at very low concentration $(1 \mathrm{nM})$ provided excellent quality images (Fig. 4 and Fig. S7, ESI $\dagger$ ) of cell plasma membranes (dSQ12S) or cytoplasm (SQ8S). Remarkably, clear plasma membrane staining with dSQ12S was observed even after $1 \mathrm{~h}$ of incubation.

In order to determine in which cytoplasmic compartment SQ8S and SQ12S accumulated, a colocalisation experiment with endoplasmic reticulum (ER)-tracker green was performed. The results showed clear colocalisation of SQ8S and SQ12S with the ER-tracker, whereas dSQ12S perfectly stained the plasma membrane with no sign of colocalisation (Fig. S8, ESI $\dagger$ ).

In sharp contrast, commercial probe DiD at $20 \mathrm{nM}$ showed no detectable fluorescence after 5-10 min (Fig. 3). Even after 1 hour, DiD failed in staining the cell membranes as dim fluorescence could only be detected in the form of dots (Fig. S9, $\mathrm{ESI}^{\dagger}$ ). It is highly probable that DiD formed insoluble aggregates that penetrated into the cells by endocytosis. Data in the literature show that membrane staining requires much higher DiD concentrations $^{15,16}$ and special delivery agents, as for instance in the case of the commercial kit Vybrant ${ }^{\mathrm{TM}}$ DiD. Our microscopy data with Vybrant ${ }^{\mathrm{TM}}$ DiD showed that at $20 \mathrm{nM}$ it failed to stain cell membranes (Fig. S10, ESI $\dagger$ ), while at $2 \mu \mathrm{M}$ (recommended in the protocol), the staining was heterogeneous, so that many cells remained unlabeled (Fig. S11, ESI $\dagger$ ). The other commercial membrane probe CellVue ${ }^{\circledR}$ Claret, which is derived from the PKH family, ${ }^{31}$ showed heterogeneous staining at $20 \mathrm{nM}$, where many cells also remained unlabeled. This uneven staining is probably related to the low concentration compared to that recommended in the protocol $(2 \mu \mathrm{M})$. Moreover, CellVue ${ }^{\circledR}$ Claret required the use of a special low salt medium "diluant C" 
and did not work in a common saline buffer such as HBSS (Fig. S12, ESI $\dagger$ ).

These results point out several key advantages of dSQ12S compared to the existing commercial fluorescent plasma membrane probes operating in the far-red to NIR regions: (a) dSQ12S works well at >1000-fold lower concentration ( $1 \mathrm{nM}$ vs. $2 \mu \mathrm{M})$; (b) it stains homogeneously all cells even at nanomolar concentrations; and (c) it can be used with usual saline buffers. Moreover, compared to FM 4-64 and DiD, dSQ12S exhibits much more specific staining of cell plasma membranes with minimal internalization. It is also at least 10-fold brighter than other high-performance membrane probes based 3-methoxyflavones, ${ }^{17}$ Nile Red, ${ }^{18}$ and BODIPY ${ }^{19,20}$ operating in shorter-wavelength spectral region. Regarding SQ8S, it was successfully used at sub-nanomolar concentration (Fig. S13, ESI $\dagger$ ), whereas the typical concentration for commercial ER trackers green and red recommended by the producer (Life Technologies) is $1 \mu \mathrm{M}$. Thus, dSQ12S and SQ8S are by far the brightest probes for cell membranes developed to date. This exceptional brightness can be explained by the molecular design that enables efficient staining of cell membranes as well as very high extinction coefficient $\left(\sim 300000 \mathrm{M}^{-1} \mathrm{~cm}^{-1}\right)$ combined with good quantum yield (50-70\%).

In conclusion, we designed three amphiphilic squaraine probes containing hydrocarbon chains and zwitterionic polar head groups. Herein, we draw the attention on the molecular design of such probes, as small modifications lead to dramatic changes in their cell permeability and therefore their distribution in the cell. SQ8S, bearing an alkyl chain and a zwitterionic group at the opposite sides of the squaraine unit, quickly crossed the plasma membrane to efficiently stain the endoplasmic reticulum membranes. SQ12S containing an anchor moiety (a chain and a zwitterion) at one end of the squaraine unit was distributed in both plasma and ER membranes. Finally, dSQ12S due to the presence of two anchors stained specifically plasma membranes without significant internalization. To the best of our knowledge, SQ8S and dSQ12S, operating well at $1 \mathrm{nM}$ concentration and compatible with twophoton excitation, are respectively the brightest molecular ER and plasma membrane fluorescent probes developed to date.

This work and RK were supported by ANR JCJC (ANR-11JS07-014-01). MC would like to dedicate this work to the viola Maestro Serge Collot (1923-2015).

\section{Notes and references}

1 B. N. G. Giepmans, S. R. Adams, M. H. Ellisman and R. Y. Tsien, Science, 2006, 312, 217-224.

2 N. C. Shaner, R. E. Campbell, P. A. Steinbach, B. N. G. Giepmans, A. E. Palmer and R. Y. Tsien, Nat. Biotechnol., 2004, 22, 1567-1572.
3 P. D. Howes, R. Chandrawati and M. M. Stevens, Science, 2014, 346, 53.

4 D. Lingwood and K. Simons, Science, 2010, 327, 46-50.

5 M. A. Model, J. L. Reese and G. C. Fraizer, Cytometry, Part A, 2009, $75,874-881$.

6 A. P. Demchenko, Y. Mély, G. Duportail and A. S. Klymchenko, Biophys. J., 2009, 96, 3461-3470.

7 A. S. Klymchenko and R. Kreder, Chem. Biol., 2014, 21, 97-113.

8 L. Jin, A. C. Millard, J. P. Wuskell, X. Dong, D. Wu, H. A. Clark and L. M. Loew, Biophys. J., 2006, 90, 2563-2575.

9 D. M. Owen, D. J. Williamson, A. Magenau and K. Gaus, Nat. Commun., 2012, 3, 1256.

10 I. Ziomkiewicz, A. Loman, R. Klement, C. Fritsch, A. S. Klymchenko, G. Bunt, T. M. Jovin and D. J. Arndt-Jovin, Cytometry, Part A, 2013, 83, 794-805.

11 L. M. Solanko, A. Honigmann, H. S. Midtiby, F. W. Lund, J. R. Brewer, V. Dekaris, R. Bittman, C. Eggeling and D. Wustner, Biophys. J., 2013, 105, 2082-2092.

12 A. Honigmann, V. Mueller, H. Ta, A. Schoenle, E. Sezgin, S. W. Hell and C. Eggeling, Nat. Commun., 2014, 5, 5412.

13 Z. Darwich, A. S. Klymchenko, D. Dujardin and Y. Mély, RSC Adv., 2014, 4, 8481-8488.

14 S. Bolte, C. Talbot, Y. Boutte, O. Catrice, N. D. Read and B. SatiatJeunemaitre, J. Microsc., 2004, 214, 159-173.

15 L. Heinrich, A.-M. Freyria, M. Melin, Y. Tourneur, R. Maksoud, J.-C. Bernengo and D. J. Hartmann, J. Biomed. Mater. Res., Part B, 2007, 81, 153-161.

16 M. G. Honig and R. I. Hume, J. Cell Biol., 1986, 103, 171-187.

17 R. Kreder, S. Oncul, O. A. Kucherak, K. A. Pyrshev, E. Real, Y. Mely and A. S. Klymchenko, RSC Adv., 2015, 5, 22899-22905.

18 O. A. Kucherak, S. Oncul, Z. Darwich, D. A. Yushchenko, Y. Arntz, P. Didier, Y. Mély and A. S. Klymchenko, J. Am. Chem. Soc., 2010, 132, 4907-4916.

19 X. Zhang, C. Wang, L. Jin, Z. Han and Y. Xiao, ACS Appl. Mater. Interfaces, 2014, 6, 12372-12379.

20 I. Lopez-Duarte, T. T. Vu, M. A. Izquierdo, J. A. Bull and M. K. Kuimova, Chem. Commun., 2014, 50, 5282-5284.

21 L. Yuan, W. Y. Lin, K. B. Zheng, L. W. He and W. M. Huang, Chem. Soc. Rev., 2013, 42, 622-661.

22 A. Ajayaghosh, Acc. Chem. Res., 2005, 38, 449-459.

23 B. Oswald, L. Patsenker, J. Duschl, H. Szmacinski, O. S. Wolfbeis and E. Terpetschmg, Bioconjugate Chem., 1999, 10, 925-931.

24 E. Arunkumar, A. Ajayaghosh and J. Daub, J. Am. Chem. Soc., 2005, 127, 3156-3164.

25 I. A. Karpenko, A. S. Klymchenko, S. Gioria, R. Kreder, I. Shulov, P. Villa, Y. Mely, M. Hibert and D. Bonnet, Chem. Commun., 2015, 51, 2960-2963.

26 I. A. Karpenko, M. Collot, L. Richert, C. Valencia, P. Villa, Y. Mely, M. Hibert, D. Bonnet and A. S. Klymchenko, J. Am. Chem. Soc., 2015, 137, 405-412.

27 J. Li, K. Guo, J. Shen, W. Yang and M. Yin, Small, 2014, 10, 1351-1360.

28 V. V. Shynkar, A. S. Klymchenko, C. Kunzelmann, G. Duportail, C. D. Muller, A. P. Demchenko, J. M. Freyssinet and Y. Mely, J. Am. Chem. Soc., 2007, 129, 2187-2193.

29 J. E. Hein and V. V. Fokin, Chem. Soc. Rev., 2010, 39, 1302-1315.

30 I. Texier, M. Goutayer, A. Da Silva, L. Guyon, N. Djaker, V. Josserand, E. Neumann, J. Bibette and F. Vinet, J. Biomed. Opt., 2009, 14, 054005.

31 P. K. Horan, M. J. Melnicoff, B. D. Jensen and S. E. Slezak, Methods Cell Biol., 1990, 33, 469-490. 